Clustering of Stearic Acids in Model Phospholipid Membranes Revealed by Double ElectronElectron Resonance

Anna S. Smorygina, ${ }^{1}$ Elena A. Golysheva, ${ }^{1}$ Sergei A. Dzuba ${ }^{1,2 *}$

${ }^{1}$ Institute of Chemical Kinetics and Combustion, Russian Academy of Sciences, Novosibirsk, Russia

${ }^{2}$ Department of Physics, Novosibirsk State University, Novosibirsk, Russia

*Author for correspondence, e-mail: dzuba@kinetics.nsc.ru

Content:

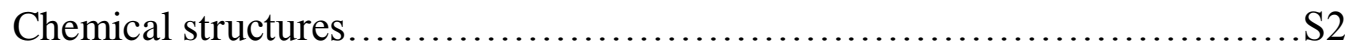

Echo-detected EPR spectrum and excitation frequencies...................S3

DEER signal correction upon the pumping pulse passage through

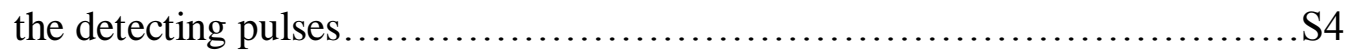

Examples of the original DEER time traces........................... S5

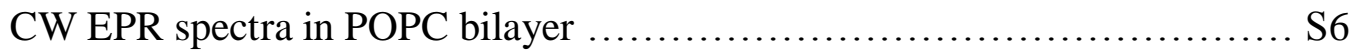

Checking orientational selectivity....................................... 7

Calibration experiment for $\mathrm{pB}$ determination..............................S8

Simulations of DEER signal decay for spin labels in two planes.............S9 


\section{Chemical structures}<smiles>CCCCCCCCC/C=C\CCCCCCCCC(=O)OC[C@H](O)COP(=O)([O-])OCC[N+](C)(C)C</smiles>

DOPC<smiles>CCCCCCCCCCCCCCCCCCCCCC(=O)OC[C@@H](COP(=O)([O-])OCCC)OCC(=O)OP(=O)(O)OCC[N+](C)(C)C</smiles>

DPPC<smiles>CCCCCCCCCCCCCCCC(=O)OC[C@H](OCCOP(=O)(O)OCC[N+](C)(C)C)OC(=O)CCCCCCCCCCC</smiles>

POPC<smiles>CCCCCCCCCCCCC1(CCCC(=O)O)CCC(C)(C)N1O</smiles>

5-DSA<smiles>CC1(C)CCC(C)(CCCCCCCCCCCCCCC(=O)O)N1[O]</smiles>

16-DSA

Scheme S1. 


\section{Echo-detected EPR spectrum and excitation frequencies}

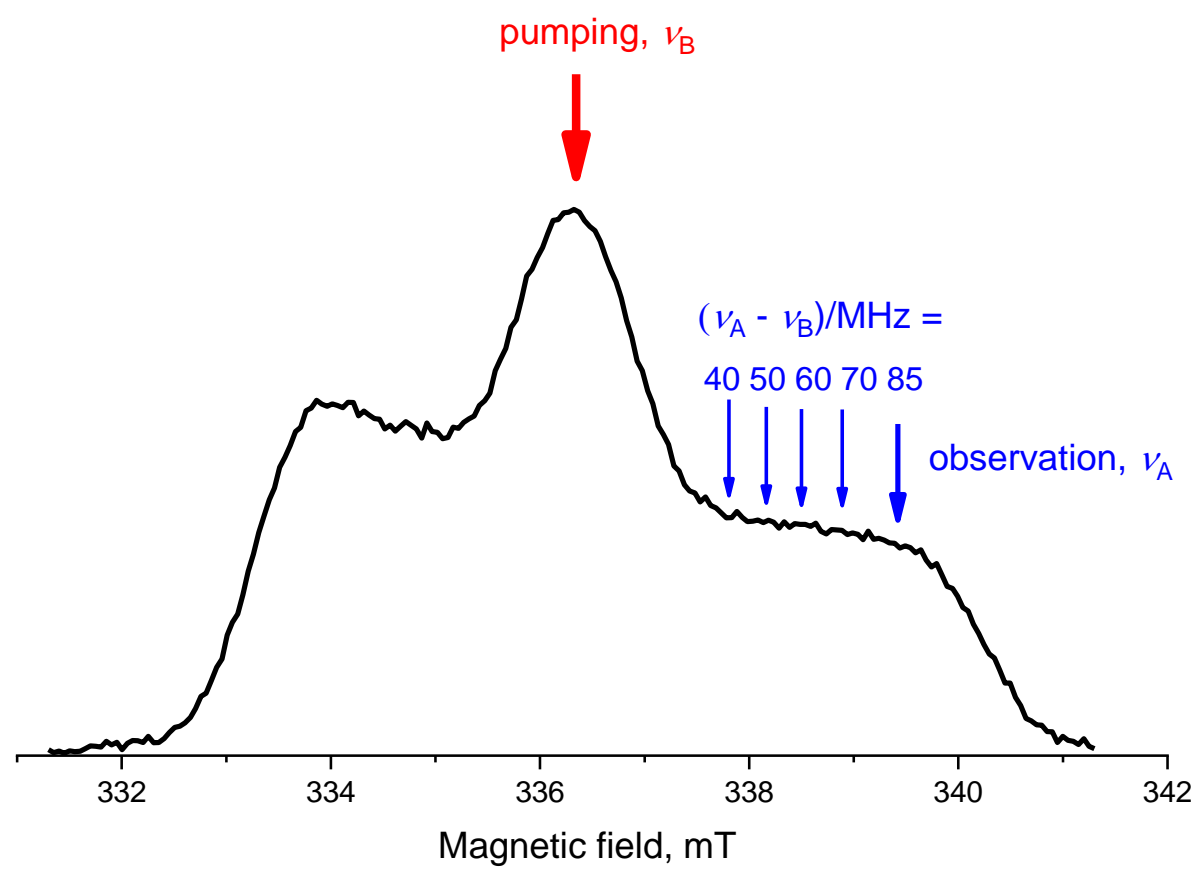

Figure S1. Echo-detected EPR spectrum obtained with scanning magnetic field and fixed time delay $\tau$ (120 ns) between two microwave pulses in the pulse sequence $(\pi / 2)_{v A}-\tau-\pi_{v A}-$ echo. The arrows show field positions for observation at microwave frequency $v_{\mathrm{A}}$ and excitation at microwave frequency $v_{\mathrm{B}}$. The sample is $0.5 \mathrm{~mol} \% 5$-DSA in the POPC bilayer, temperature $80 \mathrm{~K}$. 


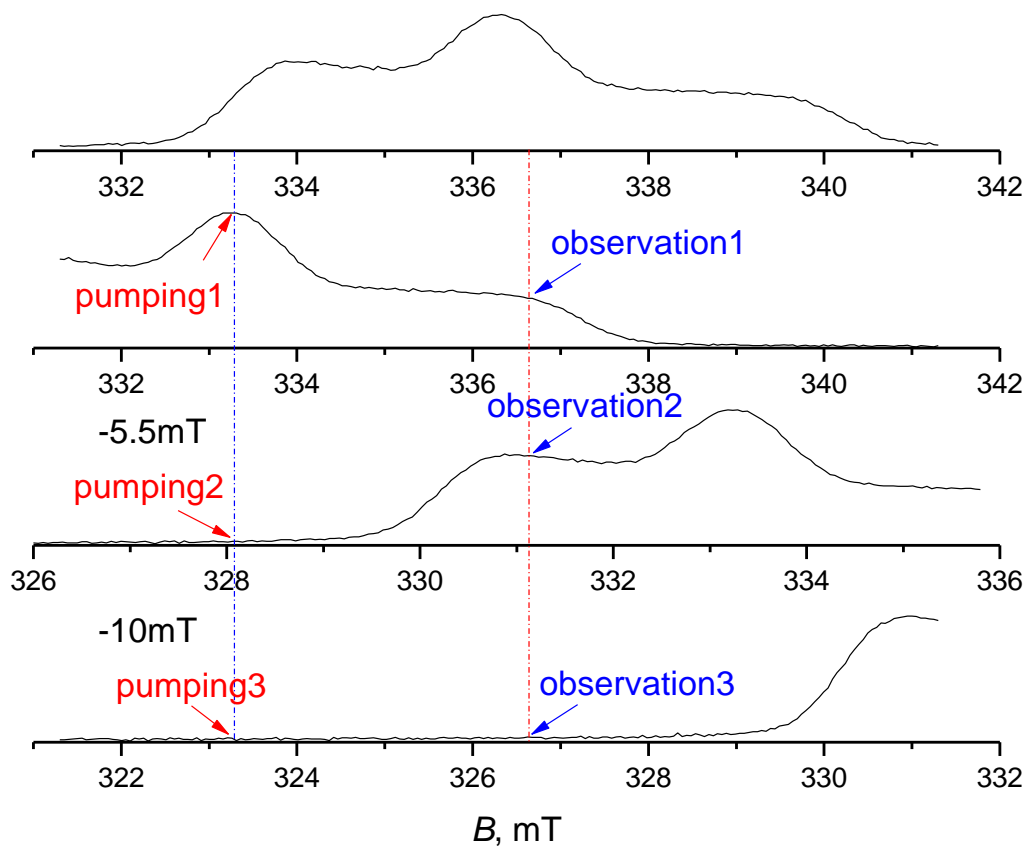

Figure S2. Calibration measurements at three different magnetic field positions.

The corrected $V(\mathrm{t})$ time dependence is obtained by relation: ${ }^{35}$

$$
V(t)=\frac{V_{1}(t)-V_{3}(t)}{V_{2}(t)-V_{3}(t)}
$$

(35) Milov, A. D.; Grishin, Y. A.; Dzuba, S. A.; Tsvetkov, Y. D. Effect of Pumping Pulse Duration on Echo Signal Amplitude in Four-Pulse PELDOR. Appl. Magn. Reson. 2011, 41, 59-67. 
Examples of the original DEER time traces

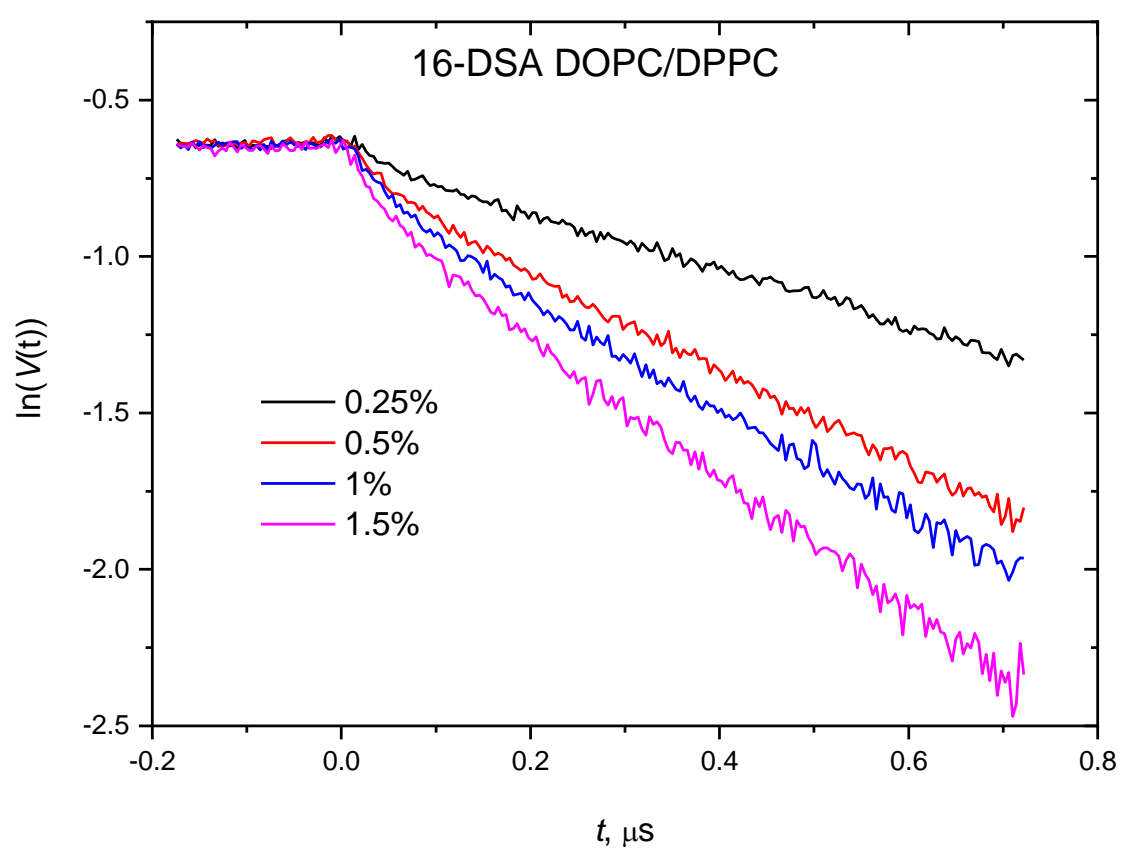

Figure S3. Original three-pulse DEER time traces for different 16-DSA content in DOPC/DPPC (1:1) bilayer. Temperature is $80 \mathrm{~K}$. 


\section{CW EPR spectra in POPC bilayer}

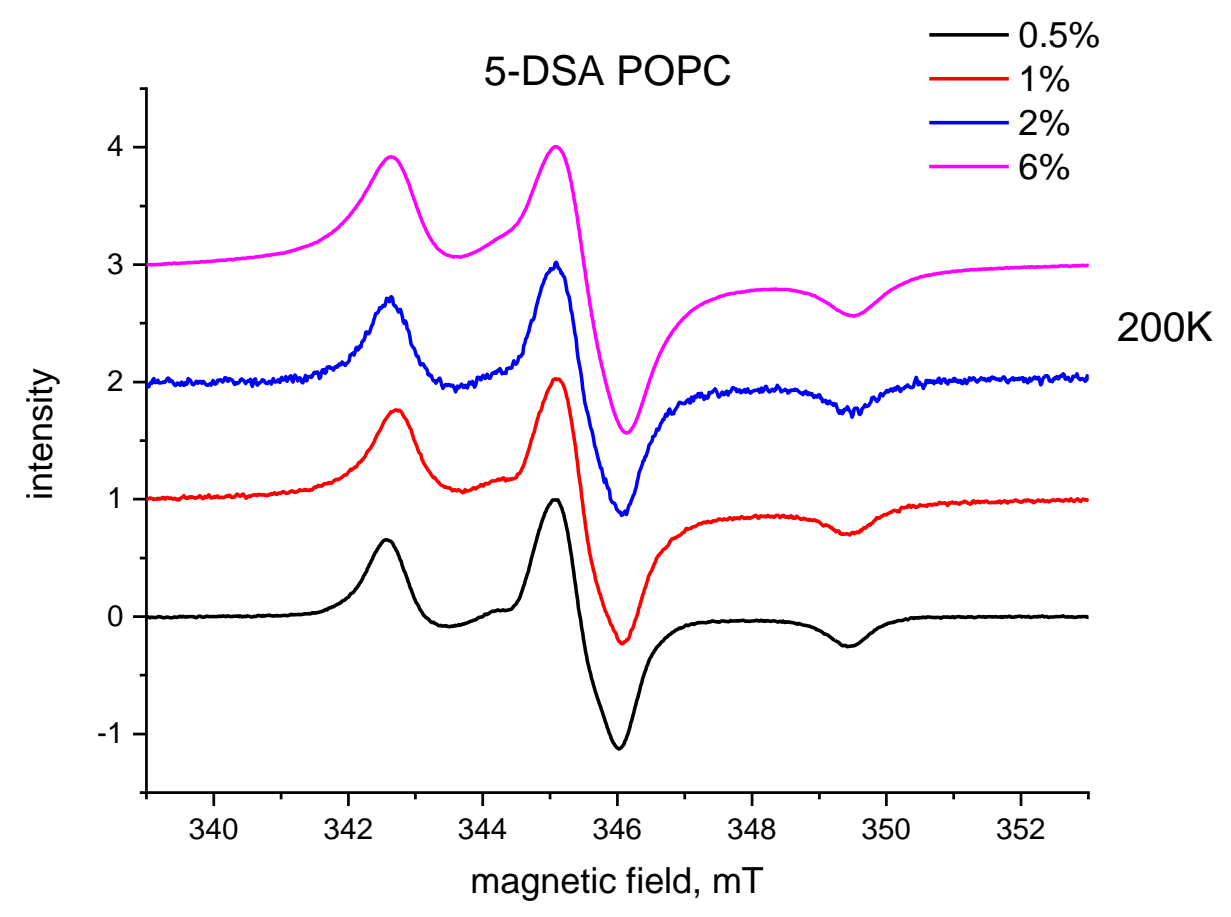

Figure S4. CW EPR spectra taken at $200 \mathrm{~K}$ for 5-DSA at different concentrations in the POPC bilayers. Spectra are normalized to the same intensity of the central component and vertically shifted. 


\section{Checking orientational selectivity}

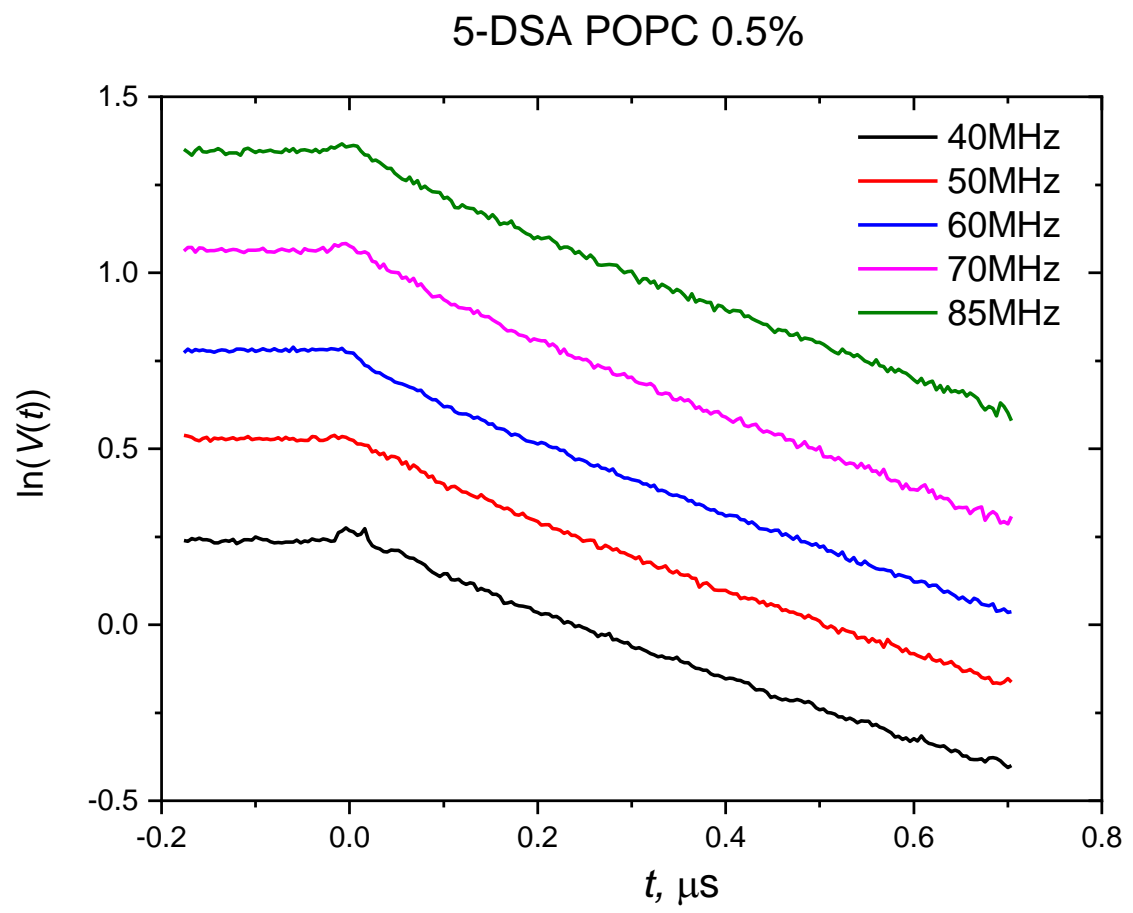

Figure S5. DEER time traces for different $v_{A}-v_{B}$ frequency offsets (see Figure S1). The sample is 0.5 mol \% 5-DSA in POPC bilayer. 


\section{Calibration experiment for $p_{B}$ determination}

The samples are $5 \mathrm{mM}$ 16-DSA and $5 \mathrm{mM}$ 5-DSA in ethanol/methanol (95:5) glass.
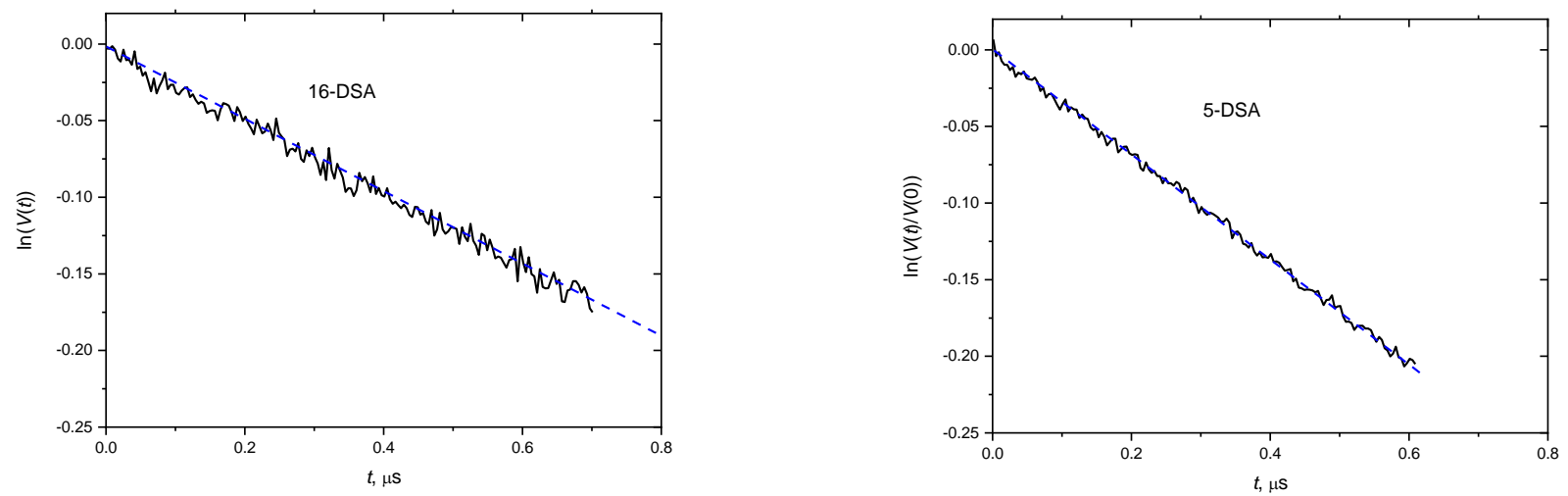

Figure S6. DEER time traces for 5mM 16-DSA (left) and 5-DSA (right) in ethanol/methanol (95:5) glass at $80 \mathrm{~K}$. The dashed straight lines present linear approximations

Using formula for uniform three-dimensional distribution

$$
V_{3 D}(t)=V(0) \exp \left(-\frac{8 \pi^{2}}{9 \sqrt{3}} \frac{g^{2} \mu_{B}^{2}}{\hbar} C p_{B} t\right),
$$

from the slope tangents we obtain $p_{B}=0.049$ for 16 -DSA and $p_{B}=0.068$ for 5-DSA. 


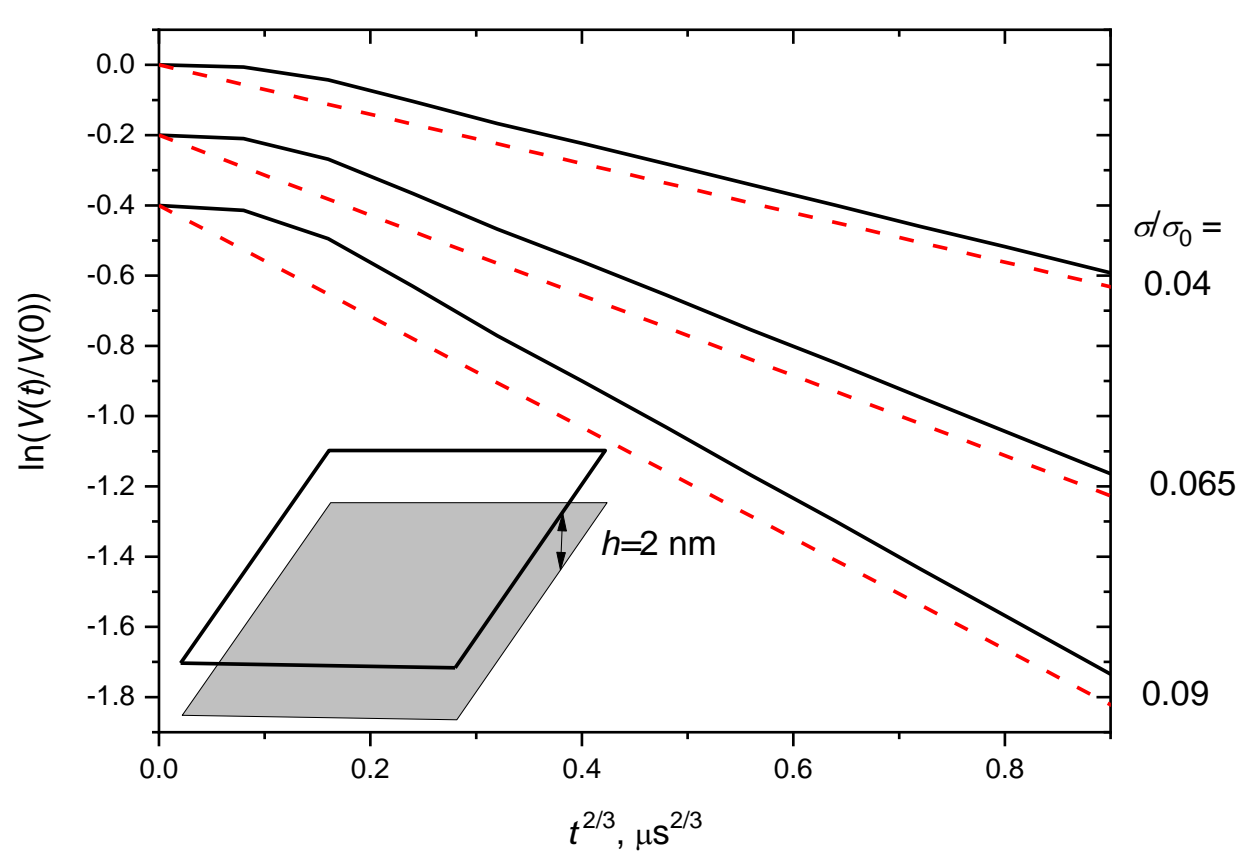

Figure S7. DEER time trace calculated ${ }^{23}$ for spins randomly distributed in two infinite parallel planes separated by the distance $h=2 \mathrm{~nm}$, for three different local concentrations. Dashed lines show the cases when $h=0$ (the two planes coincide). Curves are vertically shifted for better visualization.

(23)Kardash, M.E.; Dzuba, S.A. Lipid-mediated Clusters of Guest Molecules in Model Membranes and their Dissolving in Presence of Lipid Rafts. J. Phys. Chem. B 2017, 121, 5209-5217. 